\title{
consayronsuower
}

\section{Unkind cuts for incense}

Gold, frankincense and mymh

- the three royal gifts of the

Christmas story - remain valuable commodities. But as Toon Rijkers et al. report in the Joumal of Applied Ecology $(43,1188-1195 ; 2006)$, the latter-day story of frankincense is also a tale for our times.

Frankincense is a resin produced by several small trees of the genus Boswellia, which grow in subSaharan Africa, from Nigeria to the Horn of Africa, and Arabia and the Himalayan foothills. The resin is exuded naturally from leaves and shoots, but especially from wounds. Its natural function is probably to ward off grazers and fungal attack. But the resin's fragrance has also made itprized as a perfume, fumigant, flavouring andmedicinal compound.

Rijkers et al looked at frankincense production in Eritrea, where it is used locally for cultural and medicinal purposes, and is also exported. Wild Boswellia papyrifera trees are tapped by making incisions around the trunk of the tree, starting inmidSeptember at the end of the summer monsoon. The resin isharvested every three weeks by reopening the incisions, and harvesting continues throughout the dry season. Boswellia trees also produce their flower buds at the end of the wet season, however. Could the canstantharvest of resin, commencing at the time of floral induction, be a serious drain on the trees' carbon resources? Notleast because leaves are lost at the beginning of the dry seasan, so carbon forflowering and resin production must be obtained from storage products.

To investigate the effects of resintapping on the tree life-cycle, and especially seed quality, Rijkers et al checked the seed-germination potential of tapped and untapped

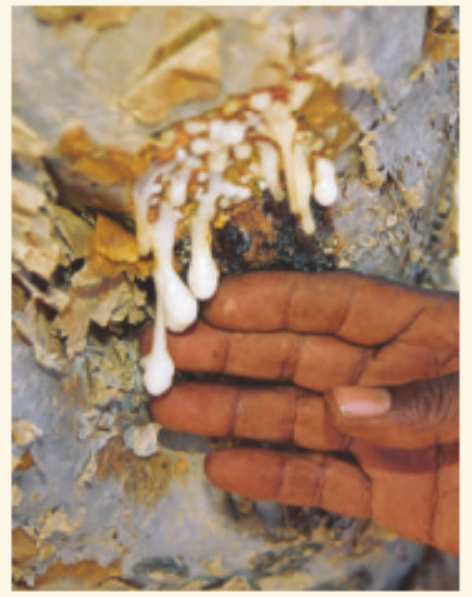

trees. They found that seeds from annually tapped trees had a germination success of only about $20 \%$ compared with a figure of $80-90 \%$ for untapped trees. Tapped trees also produced fewer inflorescences, fruits and seeds. So tapping is evidently costly to the individual tree.

There has been a severe reduction in the natural regeneration of
Boswellia in Eritrea, to which more intense tapping may be contributing. Actions that would help to reverse this trend include reducing the amount of resin taken per tree, and resting selected trees for a few seasons; the Boswellia populations also need protection from intense grazing.

Here, however, the spectre of the 'Tragedy of the Commons' arises the resources for both frankincense harvesting and grazing are freely available, and so prone to overexploitation unless the commongood is taken into account. Self-regulation in the cause of long-term sustainability is a difficult lessonto learn, as a wider world has found out from the overexploitation of fisheries.

Peter D. Moore

Peter D. Moore is in the

Department of Biochemistry, King's

College London, Franklin-Wilkins

Building, London SE1 9NH, UK. e-mail:peter.moore@kcl.ac.uk per chip, allowing ever increasing computing power and ever higher memory density.

But other applications require transistors to be sparsely distributed over large areas, and can't afford to be limited by wafer size. A flat-panel display, for example, has transistors distributed over its whole surface that switch the miniature pixels forming the image on and off. Larger screens require the distribution of transistors over larger areas, and using singlecrystal semiconductor wafers as substrates is simply not economical in this case. Instead, large-area applications use 'foreign', non-semiconductor substrates, such as metals, ceramics or glass, that can be manufactured as large sheets. In the future, substrates might also include flexible and less brittle materials such as plastic foils ${ }^{2}$.

Fabricating transistors with sufficient performance and high yield over large areas on foreign substrates is enormously challenging. Most conventional semiconductors, including silicon, can be forced to grow as defect-free films only if the growth substrate provides a perfect template for their crystal structure. When deposited on an arbitrary substrate, they tend to form amorphous, defect-infested films. Decades of research have been invested in improving the quality of these amorphous semiconductors to levels acceptable for the display industry.

One particular improvement involves recrystallizing the amorphous film into a polycrystalline film consisting of small, single-crystal grains, each with a particular crystal orientation, separated by thin boundary regions. The properties of polycrystalline materials are less ideal than those of monocrystalline materials, but are in many respects better than those of amorphous materials. But the recrystallization process requires high temperatures, so, although it can be applied with success to, say, a glass substrate, it is delicate and difficult to scale the process up to an industrial level for substrates such as plastic foil that cannot withstand elevated temperatures.

Because the transistors in large-area applications are distributed over the surface, a high-quality semiconductor film is in fact not required over the entire area. A viable way around the large-area problem could thus consist of providing semiconductor material only at those places that have to bear transistors.

Briseno et al. ${ }^{1}$ come up with an original solution to achieve precisely this. Their process hinges on a unique combination of the properties of organic semiconductors and the science of crystal nucleation and growth. First, pure crystals of so-called conjugated molecules - organic molecules that have alternate single and double covalent bonds - are known to be pretty good semiconductors 3 .

Second, because these molecules are bound into a crystal through weak van der Waals forces, rather than through stronger covalent bonds, the energy needed to form crystals from them is moderate. Such crystals can therefore be formed at low temperatures, between room temperature and a few hundred degrees Celsius, that are compatible with the processing of large-area substrates.

Finally, thermodynamics teaches us that many controllable parameters influence the energy barrier against the nucleation of crystals. These can be used to determine the location of the nudeation and growth of crystals on a surface. A known method for promoting local crystal nucleation is to 'stamp' a substrate with an extremely thin layer of molecules, known as a self-assembled monolayer, that is terminated by a chemical group with a particular affinity for the atoms or molecules of the desired crystal material ${ }^{5}$.

Briseno et $a L^{1}{ }^{1}$ use a different method that is less dependent on chemical affinity. They start by stamping an organic layer of significant roughness onto the substrate. Roughness is known to increase surface energy, and the nucleation and growth of crystals can then be steered to occur selectively on the rougher areas. The authors show that the method can be used to grow many different semiconducting conjugated molecular crystals. These include molecules that are notoriously difficult to grow as films on substrates, such as rubrene $^{6}$, and some with high vapour pressure, such as anthracene, that evaporate spontaneously in the high-vacuum environments in which thin-film deposition processes are generally conducted. But most importantly for device applications, the authors grow organic semiconductor crystals directly onto metal electrodes - forming a basic transistor - and show that these electrodes are functional after the process.

Considerable work is still needed to turn this technique into a truly viable semiconductor technology. In particular, the control of 